PROCEEDINGS OF THE

AMERICAN MATHEMATICAL SOCIETY

Volume 134, Number 7 , Pages 1927-1935

S 0002-9939(05)08193-1

Article electronically published on December 19, 2005

\title{
ENTIRE PLURICOMPLEX GREEN FUNCTIONS AND LELONG NUMBERS OF PROJECTIVE CURRENTS
}

\author{
DAN COMAN
}

(Communicated by Mei-Chi Shaw)

\begin{abstract}
Let $T$ be a positive closed current of bidimension $(1,1)$ and unit mass on the complex projective space $\mathbb{P}^{n}$. We prove that the set $V_{\alpha}(T)$ of points where $T$ has Lelong number larger than $\alpha$ is contained in a complex line if $\alpha \geq 2 / 3$, and $\left|V_{\alpha}(T) \backslash L\right| \leq 1$ for some complex line $L$ if $\alpha \geq 1 / 2$. We also prove that in dimension 2 and if $\alpha \geq 2 / 5$, then $\left|V_{\alpha}(T) \backslash C\right| \leq 1$ for some conic $C$.
\end{abstract}

\section{INTRODUCTION}

Let $T$ be a positive closed current of bidimension $(1,1)$ on the complex projective space $\mathbb{P}^{n}$, which has mass $\|T\|=1$ (see Section 2 for the definition of $\|T\|$ ). Siu's theorem [S] states that the upper level sets $E_{\alpha}(T)$ of the Lelong numbers $\nu(T, \cdot)$ of $T$,

$$
E_{\alpha}(T):=\left\{p \in \mathbb{P}^{n}: \nu(T, p) \geq \alpha\right\}, \alpha>0,
$$

are analytic subvarieties of $\mathbb{P}^{n}$ of dimension at most one. Hence by Chow's theorem, $E_{\alpha}(T)$ are algebraic varieties.

Here we consider the following upper level sets of Lelong numbers of $T$ :

$$
V_{\alpha}(T):=\left\{p \in \mathbb{P}^{n}: \nu(T, p)>\alpha\right\}, \alpha<1 .
$$

Given a finite set $S$, we denote by $|S|$ the number of points of $S$. The main results of this paper are the following theorems.

Theorem 1.1. If $\alpha \geq 1 / 2$, then there exists a complex line $L$ such that:

- either $V_{\alpha}(T) \subseteq L$,

- or $V_{\alpha}(T)$ is a finite set and $\left|V_{\alpha}(T) \backslash L\right| \leq 1$.

Moreover, if $\alpha \geq 2 / 3$, then $V_{\alpha}(T) \subseteq L$.

Theorem 1.2. If $n=2$ and $\alpha \geq 2 / 5$, then there exists a (possibly reducible) conic $C$ such that:

- either $V_{\alpha}(T) \subseteq C$,

- or $V_{\alpha}(T)$ is a finite set and $\left|V_{\alpha}(T) \backslash C\right| \leq 1$.

Received by the editors September 9, 2004 and, in revised form, February 1, 2005.

2000 Mathematics Subject Classification. Primary 32U25, 32U35; Secondary 32U05, 32U40.

Key words and phrases. Pluricomplex Green functions, Lelong numbers.

The author was supported by NSF grant DMS 0140627.

(C)2005 American Mathematical Society
Reverts to public domain 28 years from publication 
The proofs of these theorems require the construction of entire plurisubharmonic functions of logarithmic growth and with logarithmic poles in some special finite subsets of $\mathbb{C}^{n}$. In certain cases such functions were constructed in [Co and [CN].

If $D \subseteq \mathbb{C}^{n}$ is an open set, we denote by $P S H(D)$ the class of plurisubharmonic functions in $D$. A function $u \in P S H(D)$ is said to have a logarithmic pole of weight $\alpha>0$ at $p \in D$ if

$$
u(z)=\alpha \log \|z-p\|+O(1)
$$

holds for $z \in D$ near $p$. We say that $u \in P S H\left(\mathbb{C}^{n}\right)$ has logarithmic growth if

$$
\gamma_{u}:=\limsup _{\|z\| \rightarrow+\infty} \frac{u(z)}{\log \|z\|}<+\infty \text {. }
$$

Let $S$ be a finite subset of $\mathbb{C}^{n}$. Let $u \in P S H\left(\mathbb{C}^{n}\right) \cap L_{l o c}^{\infty}\left(\mathbb{C}^{n} \backslash S\right)$ have logarithmic growth. If $u=-\infty$ on $S$ and $u$ verifies the Monge-Ampère equation $\left(d d^{c} u\right)^{n}=0$ on $\mathbb{C}^{n} \backslash S$, we call $u$ an entire pluricomplex Green function of $S$.

We let $E(S)$ be the class of entire pluricomplex Green functions of $S$ with logarithmic poles of weight 1 at all points of $S$. Moreover, we let $\widetilde{E}(S) \subset P S H\left(\mathbb{C}^{n}\right)$ be the class of plurisubharmonic functions with logarithmic growth, which are locally bounded on $\mathbb{C}^{n} \backslash S$ and have logarithmic poles of weight 1 at the points of $S$. These classes were introduced in [CN], where we defined and studied two affine invariants $\gamma(S) \geq \widetilde{\gamma}(S)$ of the set $S$ :

$$
\gamma(S)=\inf \left\{\gamma_{u}: u \in E(S)\right\}, \widetilde{\gamma}(S)=\inf \left\{\gamma_{u}: u \in \widetilde{E}(S)\right\} .
$$

These numbers are connected to the singular degree of $S$ introduced in [W] and studied in $[\mathrm{W}]$ and $\mathrm{Ch}$.

We note that entire pluricomplex Green functions were generally considered in the case when they are locally bounded, for example when dealing with the extremal function of a compact set, while pluricomplex Green functions with logarithmic poles were considered mostly in the case of bounded domains, by prescribing their boundary values to be 0 (see e.g. the survey $\mathrm{B}$ ). For our purposes, it is useful to combine these two cases, and consider entire plurisubharmonic functions with finitely many logarithmic poles, as in $\mathrm{Co}$ and $\mathrm{CN}$.

Theorems 1.1 and 1.2. together with two other related results, are proved in Section 3. We also give examples of currents which show that the results of these theorems are sharp. In Proposition 2.1 from Section 2 we show the connection between the Lelong numbers of projective currents at the points of a finite set $S$, and the growth constants $\gamma_{u}$ of functions $u \in P S H\left(\mathbb{C}^{n}\right)$ with logarithmic poles in $S$. The entire pluricomplex Green functions needed to prove Theorems 1.1 and 1.2 are constructed in Section 2 .

\section{Entire Pluricomplex Green functions}

We denote by $[z: t],(z, t) \in \mathbb{C}^{n} \times \mathbb{C},(z, t) \neq(0,0)$, the homogeneous coordinates on $\mathbb{P}^{n}$, and we use the standard embedding $\mathbb{C}^{n} \equiv\left\{[z: 1] \in \mathbb{P}^{n}: z \in \mathbb{C}^{n}\right\}$. Let $\omega$ be the standard Kähler form on $\mathbb{P}^{n}$, corresponding to the Fubini-Study metric. Then

$$
\left.\omega\right|_{\mathbb{C}^{n}}=d d^{c} V, V(z):=\log \sqrt{1+\|z\|^{2}},
$$

where $d^{c}=(\partial-\bar{\partial}) /(2 \pi i)$. If $T$ is a positive closed current on $\mathbb{P}^{n}$ of bidimension $(l, l)$, its mass is given by

$$
\|T\|=\int_{\mathbb{P} n} T \wedge \omega^{l} .
$$


The following simple result is analogous to [CN, Theorem 3.4].

Proposition 2.1. Let $S=\left\{p_{1}, \ldots, p_{k}\right\} \subset \mathbb{C}^{n}$ and let $T$ be a positive closed current on $\mathbb{P}^{n}$ of bidimension $(l, l)$. If $u \in P S H\left(\mathbb{C}^{n}\right)$ has logarithmic growth, it is locally bounded outside a finite set, and $u(z) \leq \alpha_{j} \log \left\|z-p_{j}\right\|+O(1)$ for $z$ near $p_{j}$, where $\alpha_{j}>0,1 \leq j \leq k$, then

$$
\sum_{j=1}^{k} \alpha_{j}^{l} \nu\left(T, p_{j}\right) \leq \gamma_{u}^{l}\|T\|
$$

In particular, $\sum_{j=1}^{k} \nu\left(T, p_{j}\right) \leq \widetilde{\gamma}(S)^{l}\|T\|$.

Proof. Since $u$ is locally bounded outside a finite set, the positive measure $T \wedge$ $\left(d d^{c} u\right)^{l}$ is well defined on $\mathbb{C}^{n}$, by [D, Proposition 2.1]. Demailly's first comparison theorem for Lelong numbers with weights [D, Theorem 5.1] implies that

$$
T \wedge\left(d d^{c} u\right)^{l}\left(\left\{p_{j}\right\}\right) \geq \alpha_{j}^{l} \nu\left(T, p_{j}\right) .
$$

Using [CN, Proposition 3.2] we obtain

$$
\sum_{j=1}^{k} \alpha_{j}^{l} \nu\left(T, p_{j}\right) \leq \int_{\mathbb{C}^{n}} T \wedge\left(d d^{c} u\right)^{l} \leq \gamma_{u}^{l} \int_{\mathbb{C}^{n}} T \wedge\left(d d^{c} V\right)^{l} \leq \gamma_{u}^{l}\|T\| .
$$

We now construct entire pluricomplex Green functions of small growth for special finite subsets $S$ of $\mathbb{C}^{n}$. We denote by $m_{j}(S)$ the maximum number of points of $S$ which are contained in an algebraic curve of degree $j$. If $k \geq 5$, we note that $m_{1}(S)=2, m_{2}(S)=5$, hold for the generic set $S \subset \mathbb{C}^{2}$ with $|S|=k$.

Lemma 2.2. If $S \subset \mathbb{C}^{n},|S| \in\{3,4\}, m_{1}(S)=2$, then $\gamma(S)=2$.

Proof. If $S$ is contained in a two-dimensional complex plane, the lemma follows from analogous results in $\mathbb{C}^{2}[\mathrm{CN}$, Proposition 4.3]. Otherwise, since $\gamma(S)$ is an affine invariant, we can assume that $S \subset \mathbb{C}^{3} \times\{0\}$ consists of the points

$$
(0,0,0,0),(1,0,0,0),(0,1,0,0),(0,0,1,0) \text {. }
$$

Let

$$
u(z)=\frac{1}{2} \log \left(\sum_{j=1}^{3}\left|P_{j}\left(z_{1}, z_{2}, z_{3}\right)\right|^{2}+\sum_{k=4}^{n}\left|z_{k}\right|^{2}\right), z=\left(z_{1}, \ldots, z_{n}\right),
$$

where $P_{j}\left(z_{1}, z_{2}, z_{3}\right)=z_{j}\left(z_{1}+2 z_{2}+3 z_{3}-j\right)$. Then $\gamma_{u}=\gamma(S)=2$.

In the next two results, we consider the case of sets $S \subset \mathbb{C}^{2}$ with 7 or 8 elements.

Proposition 2.3. Let $S \subset \mathbb{C}^{2}$ be such that $|S|=7$ and $m_{2}(S)=5$. Then $S$ has an entire pluricomplex Green function $u$ with $\gamma_{u}=4$, such that $u$ has logarithmic poles of weight 2 at 3 of the points of $S$, and of weight 1 at the remaining 4 points of $S$.

Proof. We show that we can find 3 points of $S, \zeta_{1}, \zeta_{2}, \zeta_{3}$, with the following property: There exist two polynomials $P_{1}, P_{2}$ of degree 4 , with no common factors, such that $P_{1}(S)=P_{2}(S)=0$ and both $P_{1}, P_{2}$ vanish to second order at each point $\zeta_{j}$. By Bezout's theorem it follows that $S=\left\{P_{1}=P_{2}=0\right\}$, and the intersection numbers $\left(P_{1} \cdot P_{2}\right)_{\zeta_{j}}=4$ and $\left(P_{1} \cdot P_{2}\right)_{\zeta}=1$ at all other points $\zeta \in S$. By [CN, Theorem 4.1], the function $u=\frac{1}{2} \log \left(\left|P_{1}\right|^{2}+\left|P_{2}\right|^{2}\right)$ has the desired properties. 
Let $S=\left\{p_{1}, \ldots, p_{7}\right\}$. Note that $m_{2}(S)=5$ implies $m_{1}(S) \leq 3$. We consider the vector space $X$ of polynomials $P$ in $\mathbb{C}^{2}$ of degree at most 4 , which vanish on $S$ and vanish to second order at 3 given points of $S$, say $p_{1}, p_{2}, p_{3}$. Thus $P\left(p_{j}\right)=0$, $j=1, \ldots, 7$, and the first order partial derivatives of $P$ vanish at $p_{1}, p_{2}, p_{3}$. Since $P \in X$ verify 13 homogeneous linear equations, we have $\operatorname{dim} X \geq 15-13=2$.

Case 1. $m_{1}(S)=2, m_{2}(S)=5$. Let $C_{1}, C_{2}$ be quadratic polynomials vanishing at $p_{1}, p_{2}, p_{3}, p_{4}, p_{5}$, respectively at $p_{1}, p_{2}, p_{3}, p_{6}, p_{7}$. Then $C_{j}$ are irreducible, the conics $C_{j}=0$ are smooth algebraic curves, $C_{1}$ does not vanish at $p_{6}, p_{7}$, and $C_{2}$ does not vanish at $p_{4}, p_{5}$. Let $P_{1}=C_{1} C_{2}$ and $P_{2}$ be a polynomial of degree 4 vanishing on $S$ and to second order at $p_{1}, p_{2}, p_{3}$, such that $P_{1}, P_{2}$ are linearly independent. If $C_{1}$ divides $P_{2}$, then $P_{2}=C_{1} C$, where $C$ is a quadratic polynomial vanishing at $p_{1}, p_{2}, p_{3}, p_{6}, p_{7}$, so $C=\alpha C_{2}$. It follows that $P_{1}, P_{2}$ have no common factors.

Case 2. $m_{1}(S)=3, m_{2}(S)=5$. We may assume that $p_{1}, p_{2}, p_{3}$ lie on a complex line $l$, hence $p_{4}, p_{5}, p_{6}, p_{7}$ lie outside of $l$ and are in general position, since $m_{2}(S)=5$.

(i) Assume first that $\left\{p_{1}, p_{2}, p_{3}\right\}$ is the only 3 point subset of $S$ contained in a complex line. Let $C_{1}, C_{2}$ be irreducible quadratic polynomials vanishing at $p_{4}, p_{5}, p_{6}, p_{1}, p_{2}$, and respectively at $p_{4}, p_{5}, p_{6}, p_{3}, p_{7}$. We let $P_{1}=C_{1} C_{2}$ and continue as in Case 1.

(ii) Assume that, after relabelling if necessary the points of $S, p_{1}, p_{4}, p_{5}$ are also contained in a complex line, so $p_{2}, p_{3}, p_{6}, p_{7}$ are in general position. Let $L_{j k}$ be a linear polynomial such that $L_{j k}\left(p_{j}\right)=L_{j k}\left(p_{k}\right)=0$. We show that there exist 3 points of $S, p_{i}, p_{j}, p_{k}$, with the following property $(P): p_{i}, p_{j}, p_{k}$ are not contained in a complex line, and the polynomial $L_{i j} L_{j k} L_{k i}$ does not vanish at any of the remaining 4 points of $S$. Then we can continue as in (i), with the points $p_{4}, p_{5}, p_{6}$ replaced by $p_{i}, p_{j}, p_{k}$ (although the corresponding conics may now be reducible).

If $\left(L_{24} L_{25} L_{34} L_{35}\right)\left(p_{j}\right) \neq 0$ for $j=6,7$, then the points $p_{2}, p_{4}, p_{6}$ have the above property. Otherwise, we may assume that $L_{24}\left(p_{6}\right)=0$. Since $m_{2}(S)=5$, we have $L_{35}\left(p_{7}\right) \neq 0$. If $L_{35}\left(p_{6}\right) \neq 0$, then $p_{3}, p_{5}, p_{6}$ verify property $(P)$. Finally, we assume $L_{24}\left(p_{6}\right)=L_{35}\left(p_{6}\right)=0$. If $L_{25}\left(p_{7}\right) \neq 0$ or $L_{34}\left(p_{7}\right) \neq 0$, then the points $p_{2}, p_{5}, p_{7}$, respectively $p_{3}, p_{4}, p_{7}$, have property $(P)$. If $L_{25}\left(p_{7}\right)=L_{34}\left(p_{7}\right)=0$, then $p_{1}, p_{6}, p_{7}$ verify property $(P)$.

Proposition 2.4. Let $A \subset \mathbb{C}^{2}$ with $|A|=7, m_{1}(A) \leq 3, m_{2}(A)=6$, and let $\Gamma$ be the conic with $|A \cap \Gamma|=6$. Let $q \notin A \cup \Gamma$. Then $m_{1}(A \cup\{q\}) \leq 4$ and we have:

(i) If $m_{1}(A \cup\{q\}) \leq 3$ there exists $u \in P S H\left(\mathbb{C}^{2}\right)$ with $\gamma_{u}=3$, such that $u$ is locally bounded outside a finite set and $u(z) \leq \log \|z-p\|+O(1)$ near each point $p \in A \cup\{q\}$.

(ii) If $m_{1}(A \cup\{q\})=4$ there exists a subset $S$ of $A \cup\{q\}$ with 7 elements, which has an entire pluricomplex Green function as in the conclusion of Proposition 2.3.

Proof. Let $\left\{p_{1}\right\}=A \backslash \Gamma$, let $\left\{p_{2}, \ldots, p_{7}\right\}=A \cap \Gamma=A^{\prime}$, and let $C$ be a quadratic polynomial defining $\Gamma$. We have either $m_{1}\left(A^{\prime}\right)=2$ and $C$ is irreducible, or else $C=l_{1} l_{2}$, where $l_{j}$ are linear polynomials and $\left|\left\{l_{j}=0\right\} \cap A^{\prime}\right|=3,\left\{l_{1}=0\right\} \cap\left\{l_{2}=\right.$ $0\} \cap A=\emptyset$. In the latter case, we can assume that $p_{2}, p_{4}, p_{6}$ lie on the line $l_{1}=0$ and $p_{3}, p_{5}, p_{7}$ on the line $l_{2}=0$. In either case, the conic $\Gamma$ is smooth at the points $p_{j}, j \geq 2$.

(i) If $m_{1}(A \cup\{q\}) \leq 3$, let $L$ be a linear polynomial with $L\left(p_{1}\right)=L(q)=0$, and let $P_{1}=L C$. There exists a polynomial $P_{2}$ of degree 3 which is zero on $A \cup\{q\}$ and such that $P_{1}, P_{2}$ are linearly independent. Assume that $P_{2}=L C^{\prime}$, for some 
quadratic polynomial $C^{\prime}$. Since $L$ vanishes at most at one of the points $p_{2}, \ldots, p_{7}$, and the remaining 5 points determine $\Gamma$ uniquely, it follows that $C^{\prime}=\alpha C$, a contradiction. Similarly one shows that $C$ (or $l_{j}$ if $C$ is reducible) cannot divide $P_{2}$, so $P_{1}, P_{2}$ have no common factors. We let $u=\frac{1}{2} \log \left(\left|P_{1}\right|^{2}+\left|P_{2}\right|^{2}\right)$.

(ii) If $m_{1}(A \cup\{q\})=4$, then the complex line determined by $p_{1}, q$ must contain two points of $\Gamma$. After relabelling points, we can assume that $p_{1}, p_{2}, p_{3}, q$ are contained in a complex line. Let $L_{j k}$ be a linear polynomial such that $L_{j k}\left(p_{j}\right)=$ $L_{j k}\left(p_{k}\right)=0$. Since $m_{1}(A) \leq 3$, we have for each $j \geq 2$ that $L_{1 j}$ can vanish at most at one other point $p_{k}(k \geq 2, k \neq j)$.

Case 1. If some polynomial $L_{1 j}, j \geq 4$, does not vanish at any other point $p_{k}$, then there must exist another polynomial $L_{1 i}, i \geq 4$, with the same property. Note that when $C=l_{1} l_{2}$, such $i$ exists so that $p_{i}, p_{j}$ are not both contained in a line $l_{k}=0$. We let $S=A, P_{1}=L_{1 i} L_{1 j} C$, and $P_{2}$ be a polynomial of degree 4 vanishing on $S$ and to second order at $p_{1}, p_{i}, p_{j}$, such that $P_{1}, P_{2}$ are linearly independent. A direct application of Bezout's theorem shows that $P_{1}, P_{2}$ have no common factors. By the considerations at the beginning of the proof of Proposition 2.3. $u=\frac{1}{2} \log \left(\left|P_{1}\right|^{2}+\left|P_{2}\right|^{2}\right)$ is the desired pluricomplex Green function of $S$.

Case 2. If each polynomial $L_{1 j}, j \geq 4$, vanishes at some other point $p_{k}, k \geq 4$, then after relabelling points we have $L_{14}\left(p_{5}\right)=L_{16}\left(p_{7}\right)=0$. If $S=(A \cup\{q\}) \backslash\left\{p_{2}\right\}$, then $m_{2}(S)=5$, and the conclusion follows from Proposition 2.3. Indeed, the points $p_{3}, \ldots, p_{7}$ determine $\Gamma$, which does not contain $p_{1}, q$, and $p_{1}, p_{4}, p_{5}, p_{6}, p_{7}$ determine the conic $L_{45} L_{67}=0$, which does not contain $p_{3}, q$. If $S \backslash\left\{p_{j}\right\}, j \geq 4$, is contained in a conic and without loss of generality $j=4$, then this conic is defined by $L_{23} L_{67}=0$. But $L_{23} L_{67}\left(p_{5}\right) \neq 0$.

\section{UPPER LEVEL SETS OF LELONG NUMBERS}

Throughout this section, $T$ is a positive closed current of bidimension $(1,1)$ on $\mathbb{P}^{n}$, normalized by $\|T\|=1$. Then the Lelong numbers $\nu(T, p) \leq 1$ at every point $p \in \mathbb{P}^{n}$. We will need the following simple lemma:

Lemma 3.1. If $S$ is an at most countable subset of $\mathbb{P}^{n}$, then there exists a hyperplane $H$ which does not intersect $S$.

Proof. We show by induction on $k, 0 \leq k \leq n-1$, that there exists a $k$-dimensional plane $P_{k}$ which does not intersect $S$. This is clear for $k=0$, and assume such $P_{k}$ exists for $k<n-1$. The family of $(k+1)$-dimensional planes $P$ which contain $P_{k}$ is uncountable, and the sets $P \backslash P_{k}$ are pairwise disjoint. Since $S$ is at most countable and does not intersect $P_{k}$, it follows that there is a $(k+1)$-dimensional plane $P_{k+1} \supset P_{k}$ which does not intersect $S$.

Recall that for a finite subset $S$ of $\mathbb{P}^{n}, m_{j}(S)$ denotes the maximum number of points in $S$ which are contained in an algebraic curve of degree $j$. We now proceed with the proofs of Theorems 1.1 and 1.2 ,

Proof. Proof of Theorem 1.1Let $\alpha \geq 2 / 3$. Assume that some set $E_{\beta}(T), \beta>\alpha$, has dimension one, so it contains an algebraic curve $C$. By a result of $[\mathrm{S}, T=\beta[C]+R$, where $[C]$ is the current of integration along $C$ and $R$ is a $(1,1)$ bidimensional positive closed current on $\mathbb{P}^{n}$. The degree of $C$ (see e.g. [LG]) is $\|[C]\| \leq 1 / \beta<2$, so $C$ is a complex line. Since $\nu(R, p) \leq\|R\|=1-\beta<1 / 3$ at all $p \in \mathbb{P}^{n}$, it follows that $V_{\alpha}(T)=C$. 
If all sets $E_{\beta}(T), \beta>\alpha$, have dimension 0 , then $V_{\alpha}(T)$ is at most countable. By Lemma 3.1 there is a hyperplane $H$ so that $V_{\alpha}(T) \subset \mathbb{P}^{n} \backslash H$, so we may assume $V_{\alpha}(T) \subset \mathbb{C}^{n}$. If $V_{\alpha}(T)$ is not contained in a complex line, there exists $S \subseteq V_{\alpha}(T)$ with $|S|=3$ and $m_{1}(S)=2$, so $\gamma(S)=2$ by Lemma 2.2. Proposition 2.1 yields the following contradiction:

$$
3 \alpha<\sum_{p \in S} \nu(T, p) \leq \gamma(S)\|T\|=2 .
$$

We assume now $1 / 2 \leq \alpha<2 / 3$. Arguing as above, we have that either $V_{\alpha}(T)$ is a complex line, or it is at most countable and contained in $\mathbb{C}^{n}$.

If $V_{\alpha}(T)$ is countable, then $E_{1 / 2}(T)$ must contain an algebraic curve $C$. By $\underline{\underline{S}}$, $T=\frac{1}{2}[C]+R$ and the degree of $C$ is at most 2. If $C$ has degree 2 , then $R=0$, so $\nu(T, p)>1 / 2$ only at the singular points of $C$, and $V_{1 / 2}(T)$ is a finite set. We conclude that $C$ is a complex line. Since $\|R\|=1 / 2$, we have $V_{\alpha}(T) \subset C$.

We assume finally that $V_{\alpha}(T)$ is a finite set not contained in a complex line and with at least 4 elements. So there exists $S=\left\{p_{1}, p_{2}, p_{3}, p_{4}\right\} \subset V_{\alpha}(T)$ such that $p_{1}, p_{2}, p_{3}$ are not contained in a complex line. If $m_{1}(S)=2$, then Lemma 2.2 and Proposition 2.1 imply $4 \alpha<2$, a contradiction. So, after relabelling points, $p_{4}$ lies on the complex line $L$ determined by $p_{1}, p_{2}$. If there exists $p \in V_{\alpha}(T) \backslash L, p \neq p_{3}$, then at least one of the sets $S$,

$$
\left\{p_{1}, p_{2}, p_{3}, p\right\},\left\{p_{1}, p_{4}, p_{3}, p\right\},\left\{p_{2}, p_{4}, p_{3}, p\right\},
$$

has $m_{1}(S)=2$, and we reach a contradiction as above. Therefore $\left|V_{\alpha}(T) \backslash L\right|=$ 1.

Remark 3.2. The proof of Theorem 1.1 shows that if $\alpha \geq 1 / 2$ and the set $V_{\alpha}(T)$ is infinite, then $V_{\alpha}(T)$ is either a complex line or a countable subset of a complex line.

Proof. Proof of Theorem 1.2 As in the previous proof, there are two possibilities: some set $E_{\beta}(T), \beta>\alpha$, contains an algebraic curve $C$, or $V_{\alpha}(T)$ is at most countable and contained in $\mathbb{C}^{2}$.

In the first case, we have by $\underline{\underline{S}}$ that $T=\beta[C]+R$, for some positive closed $(1,1)$ current $R$ on $\mathbb{P}^{2}$, and the degree of $C$ is at most 2 . If $C$ is a conic, then $\|R\|<1 / 5$ and $V_{\alpha}(T)=C$. If $C$ is a complex line and $R=0$, then $V_{\alpha}(T)=C$. Otherwise $0<\|R\|=1-\beta<3 / 5$ and $V_{\alpha}(T)=C \cup V_{\alpha}(R)$. Since $\alpha /\|R\| \geq 2 / 3, V_{\alpha}(R)$ is a complex line or an at most countable subset of a complex line, by Theorem 1.1.

We assume next that $V_{\alpha}(T)$ is countable, so $E_{2 / 5}(T)$ contains an algebraic curve $C$ of degree at most 2 and $T=\frac{2}{5}[C]+R$. If $C$ is a conic, then $V_{\alpha}(T) \subset C$. If $C$ is a complex line, then $\|R\|=3 / 5$, so $V_{\alpha}(R)$ is contained in a complex line since $\alpha /\|R\| \geq 2 / 3$ and $V_{\alpha}(T) \subset C \cup V_{\alpha}(R)$.

We finally assume that $V_{\alpha}(T)$ is a finite set and $\left|V_{\alpha}(T) \backslash C\right|>1$, for any conic $C$. Then $V_{\alpha}(T)$ has a subset $A$ with $|A|=7$. We have the following possibilities:

Case 1. $m_{2}(A)=5$. Then $A$ has an entire pluricomplex Green function $u$ as in Proposition 2.3. Proposition 2.1 applied to $u$ and $T$ implies $10 \alpha<4$, a contradiction.

Case 2. $m_{1}(A) \leq 3, m_{2}(A)=6$. Let $C$ be the conic with $|A \cap C|=6$. Since $\left|V_{\alpha}(T) \backslash C\right|>1$, there is $q \in V_{\alpha}(T) \backslash(A \cup C)$. Applying Proposition 2.1 with the functions provided by Proposition 2.4 yields $8 \alpha<3$, or $10 \alpha<4$, a contradiction. 
Case 3. $m_{1}(A)=2$ and $A$ is contained in a conic $C$. There exists $q \in V_{\alpha}(T) \backslash C$. If $p \in A$, then $S=(A \cup\{q\}) \backslash\{p\}$ verifies the hypotheses of Case 2 .

Case 4. $m_{1}(A) \geq 3$. We show that $V_{\alpha}(T)$ has a subset $S$ with $|S|=7, m_{1}(S) \leq 3$, $m_{2}(S) \leq 6$, so we are back to Case 1 or Case 2 . Let $L_{j k}$ be the complex line determined by the points $p_{j}, p_{k}$. Let $p_{1}, p_{2}, p_{3}$ be points of $A$ so that $p_{3} \in L_{12}$. As $\left|V_{\alpha}(T) \backslash C\right|>1$ for any conic $C$, there exist points $p_{4}, \ldots, p_{7} \in V_{\alpha}(T) \backslash L_{12}$, with $p_{6}, p_{7} \notin L_{45}$. Let $S_{1}=\left\{p_{1}, \ldots, p_{7}\right\}$. Then $m_{2}\left(S_{1}\right) \leq 6$, so $m_{1}\left(S_{1}\right) \leq 4$. If $m_{1}\left(S_{1}\right) \leq 3$ we let $S=S_{1}$. If $m_{1}\left(S_{1}\right)=4$, then, after relabelling points, $p_{6}, p_{7} \in L_{14}$. Hence there exists $p_{8} \in V_{\alpha}(T) \backslash\left(L_{12} \cup L_{14}\right), p_{8} \neq p_{5}$. The point $p_{8}$ can lie on at most one of the lines $L_{5 j}, j=4,6,7$. If $p_{8} \in L_{5 j}$ for some $j \in\{4,6,7\}$, we let $S=\left(S_{1} \cup\left\{p_{8}\right\}\right) \backslash\left\{p_{j}\right\}$. Otherwise, we let $S=\left(S_{1} \cup\left\{p_{8}\right\}\right) \backslash\left\{p_{7}\right\}$.

Remark 3.3. The proof of Theorem 1.2 shows that if $n=2, \alpha \geq 2 / 5$ and the set $V_{\alpha}(T)$ is infinite, then $V_{\alpha}(T)$ is one of the following: a conic, the union of a complex line and an at most countable subset of a complex line, a countable subset of a conic.

It is not difficult to see that all cases described in Theorems 1.1 and 1.2 can occur. Here we present a few examples in $\mathbb{P}^{2}$.

Example 3.4. Let $L_{1}, L_{2}, L_{3}$ be complex lines with $L_{1} \cap L_{2} \cap L_{3}=\emptyset$, and let $\left\{p_{1}\right\}=L_{2} \cap L_{3},\left\{p_{2}\right\}=L_{1} \cap L_{3},\left\{p_{3}\right\}=L_{1} \cap L_{2}$. If $T=\frac{1}{3} \sum_{j=1}^{3}\left[L_{j}\right]$, then $E_{2 / 3}(T)=\left\{p_{1}, p_{2}, p_{3}\right\}$ is not contained in a complex line, so the value $\alpha=2 / 3$ in Theorem 1.1 is the best possible.

Example 3.5. Let $L_{1}, \ldots, L_{4}$ be complex lines so that no 3 of them pass through the same point, let $\left\{p_{j k}\right\}=L_{j} \cap L_{k}, 1 \leq j<k \leq 4$, and let $S=\left\{p_{j k}\right\},|S|=6$. If $T=\frac{1}{4} \sum_{j=1}^{4}\left[L_{j}\right]$, then $E_{1 / 2}(T)=S$ has $m_{1}(S)=3$, so the value $\alpha=1 / 2$ in Theorem 1.1] is sharp. Moreover, $E_{1 / 2}(T)$ is not contained in a conic.

Example 3.6. If $C$ is a conic and $T=[C] / 2$, then $E_{1 / 2}(T) \backslash L$ is an uncountable set, for every complex line $L$.

Example 3.7. Let $L_{j}$ be complex lines containing the point $q, L$ be a complex line with $q \notin L$, and $\left\{p_{j}\right\}=L \cap L_{j}$. If $m \geq 2$ and

$$
T=\frac{m-1}{2 m}[L]+\frac{m+1}{2 m^{2}} \sum_{j=1}^{m}\left[L_{j}\right],
$$

then $V_{1 / 2}(T)=\left\{p_{1}, \ldots, p_{m}, q\right\}$ has $m$ points on $L$.

Example 3.8. Let $L_{j}, p_{j}$ be as in Example 3.4 and let $p_{4} \notin L_{1} \cup L_{2} \cup L_{3}$. Let $L_{4}, L_{5}, L_{6}$ be the complex lines determined by the points $p_{4}$ and respectively $p_{1}, p_{2}, p_{3}$, and let $\left\{p_{5}\right\}=L_{1} \cap L_{4},\left\{p_{6}\right\}=L_{2} \cap L_{5},\left\{p_{7}\right\}=L_{3} \cap L_{6}$. Finally, let $l_{1}, l_{2}, l_{3}$ be the complex lines determined by pairs of points from $\left\{p_{5}, p_{6}, p_{7}\right\}$. If $S=\left\{p_{1}, \ldots, p_{7}\right\}$ and

$$
T=\frac{1}{15}\left(2 \sum_{j=1}^{6}\left[L_{j}\right]+\sum_{j=1}^{3}\left[l_{j}\right]\right),
$$

then $m_{2}(S)=5, E_{2 / 5}(T)=S$, so the value $\alpha=2 / 5$ in Theorem 1.2 is sharp. 
Example 3.9. Let $C$ be a conic and let $p_{j} \in C, j \geq 0$, be distinct points so that $p_{j} \rightarrow p_{0}$ as $j \rightarrow \infty$. Let $L_{j}$ be a complex line passing through $p_{j}$ and some given point $q \notin C$. If $\epsilon_{j}>0, \sum_{j=0}^{\infty} \epsilon_{j}=1 / 5$, and

$$
T=\frac{2}{5}[C]+\sum_{j=0}^{\infty} \epsilon_{j}\left[L_{j}\right]
$$

then $V_{2 / 5}(T)$ is a countable subset of $C$.

We saw in Examples 3.5 and 3.6 that for $\beta<1 / 2$ one can have $\left|V_{\beta}(T) \backslash L\right|>1$ for every complex line $L$. But if $T$ has "large" Lelong numbers at two points, then the conclusion of Theorem 1.1 still holds for some values $\beta<1 / 2$.

Theorem 3.10. Assume that $\alpha>1 / 2$ and there are points $q_{1}, q_{2} \in \mathbb{P}^{n}$ so that $\nu\left(T, q_{j}\right) \geq \alpha, j=1,2$. If $\beta=(2-\alpha) / 3$, then $\left|V_{\beta}(T) \backslash L\right| \leq 1$ for some complex line $L$ which contains at least one of the points $q_{1}, q_{2}$.

Proof. Let $L_{1}$ be the complex line determined by $q_{1}, q_{2}$. If $\left|V_{\beta}(T) \backslash L_{1}\right|>1$, let $p_{1}, p_{2} \in V_{\beta}(T) \backslash L_{1}$ and let $L_{2}$ be the complex line determined by $p_{1}, p_{2}$. We choose $1 / 2<\alpha^{\prime}<\alpha$ so that $\nu\left(T, p_{j}\right)>\left(2-\alpha^{\prime}\right) / 3$ and we consider the current

$$
R=\frac{2 \alpha^{\prime}-1}{2 \alpha^{\prime}}\left[L_{2}\right]+\frac{1}{2 \alpha^{\prime}} T \text {. }
$$

Then $\nu\left(R, q_{j}\right) \geq \alpha /\left(2 \alpha^{\prime}\right)>1 / 2$ and

$$
\nu\left(R, p_{j}\right)>\frac{2 \alpha^{\prime}-1}{2 \alpha^{\prime}}+\frac{2-\alpha^{\prime}}{6 \alpha^{\prime}}>\frac{1}{2}
$$

for $j=1,2$. Theorem 1.1 implies that one of the points $q_{1}, q_{2}$, say without loss of generality $q_{1}$, lies on $L_{2}$. We show that $V_{\beta}(T) \backslash\left\{q_{2}\right\} \subseteq L_{2}$. If not, there exists $p_{3} \in V_{\beta}(T) \backslash\left\{q_{2}\right\}, p_{3} \notin L_{2}$. If $L_{j k}$ denotes the complex line determined by $p_{j}, p_{k}$, let

$$
R=\frac{2 \alpha^{\prime}-1}{6 \alpha^{\prime}}\left(\left[L_{12}\right]+\left[L_{23}\right]+\left[L_{13}\right]\right)+\frac{1}{2 \alpha^{\prime}} T,
$$

where $1 / 2<\alpha^{\prime}<\alpha$ is such that $\nu\left(T, p_{j}\right)>\left(2-\alpha^{\prime}\right) / 3$ for $j=1,2,3$. Then $\nu\left(R, q_{j}\right)>1 / 2$ and

$$
\nu\left(R, p_{j}\right)>\frac{2\left(2 \alpha^{\prime}-1\right)}{6 \alpha^{\prime}}+\frac{2-\alpha^{\prime}}{6 \alpha^{\prime}}=\frac{1}{2} .
$$

If $S=\left\{p_{1}, p_{2}, p_{3}, q_{1}, q_{2}\right\}$, then $m_{1}(S)=3$, which contradicts Theorem 1.1

Example 3.11. Let $L_{1}, L_{2}$ be complex lines in $\mathbb{P}^{n}$ which intersect at point $q$, and let $T=\frac{1}{2}\left(\left[L_{1}\right]+\left[L_{2}\right]\right)$. Then $\nu(T, q)=1$ and $E_{1 / 2}(T) \backslash L$ is uncountable for every complex line $L$. So the assumption in Theorem 3.10 on the existence of two points where $T$ has large Lelong numbers is necessary.

Our last result shows that the complex lines from the conclusion of Theorem 1.1 are determined by 3 points where $T$ has "small" Lelong numbers.

Proposition 3.12. Let $\alpha \geq 1 / 2$ and assume that $E_{1-\alpha}(T)$ contains the points $p_{1}, p_{2}, p_{3}$ which lie on a complex line $L \subset \mathbb{P}^{n}$. Then $\left|V_{\alpha}(T) \backslash L\right| \leq 1$. Moreover, if $\alpha \geq 2 / 3$, then either $V_{\alpha}(T) \subseteq L$ or else $\left|V_{\alpha}(T)\right| \leq 2$. 
Proof. If $\left|V_{\alpha}(T) \backslash L\right|>1$, let $q_{1}, q_{2} \in V_{\alpha}(T) \backslash L$, and let $\alpha^{\prime}>\alpha$ be chosen so that $\nu\left(T, q_{j}\right)>\alpha^{\prime}, j=1,2$. Then

$$
R=\frac{2 \alpha^{\prime}-1}{2 \alpha^{\prime}}[L]+\frac{1}{2 \alpha^{\prime}} T
$$

has Lelong numbers larger than $1 / 2$ at the points of the set $S=\left\{p_{1}, p_{2}, p_{3}, q_{1}, q_{2}\right\}$. As $m_{1}(S)=3$, this contradicts Theorem 1.1 .

We assume next that $\alpha \geq 2 / 3$ and there exists $q \in V_{\alpha}(T) \backslash L$. By what was already proved, we have $V_{\alpha}(T) \backslash\{q\} \subseteq L$. Theorem 1.1 implies that $V_{\alpha}(T) \subseteq L^{\prime}$, where $L^{\prime}$ is a complex line containing $q$. It follows that $\left|V_{\alpha}(T)\right| \leq 2$.

\section{REFERENCES}

[B] E. Bedford, Survey of pluripotential theory, in Several Complex Variables: Proceedings of the Mittag-Leffler Institut 1987-1988, J.-E. Fornæss (ed.), Math. Notes 38, Princeton Univ. Press, 1993, 48-97. MR1207855 (94b:32014)

[Ch] G. V. Chudnovsky, Singular points on complex hypersurfaces and multidimensional Schwarz lemma, in Seminar on Number Theory, Paris 1979/80, Birkhäuser, 1981, 29-69. MR0633888 (83m:32002)

[Co] D. Coman, Certain classes of pluricomplex Green functions on $\mathbb{C}^{n}$, Math. Z. 235 (2000), 111-122. MR1785074 (2001i:32052)

[CN] D. Coman and S. Nivoche, Plurisubharmonic functions with singularities and affine invariants for finite sets in $\mathbf{C}^{n}$, Math. Ann. 322 (2002), 317-332. MR1893919 (2003b:32035)

[D] J. P. Demailly, Monge-Ampère operators, Lelong numbers and intersection theory, in Complex analysis and geometry, Plenum, New York, 1993, 115-193. MR1211880 (94k:32009)

[LG] P. Lelong and L. Gruman, Entire functions of several complex variables, Springer-Verlag, 1986. MR0837659 (87j:32001)

[S] Y. T. Siu, Analyticity of sets associated to Lelong numbers and the extension of closed positive currents, Invent. Math. 27 (1974), 53-156. MR0352516 (50:5003)

[W] M. Waldschmidt, Propriétés arithmétiques de fonctions de plusieurs variables (II), in Séminaire P. Lelong (Analyse), 1975/76, Lecture Notes Math. 578, Springer, 1977, 108135. MR0453659 (56:11919)

Department of Mathematics, 215 Carnegie Hall, Syracuse University, Syracuse, New YORK 13244-1150

E-mail address: dcoman@@syr.edu 